\section{Uso de medicamentos potencialmente inapropriados por idosos do Município de São Paulo, Brasil: Estudo SABE}

\author{
Use of potentially inappropriate medication by the \\ elderly in São Paulo, Brazil: SABE Study
}

\author{
Uso de medicamentos potencialmente \\ inapropiados para los adultos mayores de \\ São Paulo, Brasil: Estudio SABE
}

\begin{abstract}
Various medicines are considered unsuitable for older adults due to alterations in pharmacokinetics/pharmacodynamics, lack of therapeutic efficacy, and/or increased risk of adverse events exceeding potential benefits. The aim of this study was to determine the prevalence of potentially inappropriate medication among older adults in the city of São Paulo, Brazil. A population-based cross-sectional study was conducted in a sample of 1,254 individuals aged 60 years or older (participants in the SABE Study), representing the elderly population of the city in 2006. Potentially inappropriate medication was defined according to the Beers criteria. Prevalence of potentially inappropriate medication was 28\%. Potentially inappropriate medication was associated with polypharmacy ( $p=0.001)$, two or more diseases $(p=0.011)$, and female gender $(p=0.007)$. Thirty-six potentially inappropriate medicines were identified, the majority of which involving prescription medications. Specific criteria are needed for the Brazilian population, in addition to awareness-raising on clinical pharmacology in older adults. Prescription protocols and software programs could assist in the process of rational prescribing in this age group.
\end{abstract}

Drug Utilization; Aged; Pharmacoepidemiology
Teresa Cristina Jahn Cassoni 1

Ligiana Pires Corona 1

Nicolina Silvana Romano-Lieber 1

Silvia Regina Secoli 2

Yeda Aparecida de Oliveira Duarte 2

Maria Lúcia Lebrão ${ }^{1}$

\section{Resumo}

Alguns medicamentos são considerados impróprios para o idoso, devido a alterações na farmacocinética e farmacodinâmica, por falta de eficácia terapêutica ou por um risco aumentado de efeitos adversos superando seus benefícios. Verificou-se a prevalência do uso de medicamentos potencialmente inapropriados, segundo os critérios de Beers, por idosos do Município de São Paulo, Brasil, participantes do Estudo SABE. Trata-se de um estudo transversal, de base populacional, cuja amostra de 1.254 indivíduos com 60 anos ou mais representava a população idosa do município no ano de 2006. Verificou-se a prevalência de $28 \%$ de uso de medicamentos potencialmente inapropriados. No modelo de regressão logística múltipla, as variáveis associadas ao uso foram uso de cinco medicamentos ou mais ( $p=0,001)$, presença de duas ou mais doenças ( $p=0,011)$ e sexo feminino ( $p=0,007)$. Identificou-se o uso de 36 medicamentos potencialmente inapropriados, a maioria de venda sob prescrição. São necessários critérios específicos para a população brasileira e ampla divulgação da farmacologia clínica do idoso. Protocolos ou softwares para prescrição também podem auxiliar a prescrição racional para esse grupo.

Uso de Medicamentos; Idoso; Farmacoepidemiologia 


\section{Introdução}

Indivíduos idosos apresentam respostas a medicamentos diferentes daquelas apresentadas por pessoas mais jovens, devido a alterações farmacocinéticas e farmacodinâmicas próprias do envelhecimento. Nesse contexto, alguns são considerados medicamentos potencialmente inapropriados para esse grupo etário, seja por falta de evidências acerca da eficácia terapêutica, pelo risco aumentado de eventos adversos que supera os benefícios, quando existe uma alternativa terapêutica mais segura ou quando o uso do medicamento pode agravar doenças preexistentes do idoso 1,2,3,4.

Diante dessa perspectiva, nos países desenvolvidos, a partir de meados da década de 1980 e, principalmente, da década de 1990, a preocupação com os efeitos prejudiciais do uso de medicamentos por idosos impulsionou prescritores, farmacêuticos e pesquisadores a desenvolver e aplicar diversos métodos e instrumentos para identificar padrões inadequados de prescrição e problemas farmacoterapêuticos envolvendo esse grupo populacional 5 .

Critérios explícitos são úteis para avaliar o uso de medicamentos por idosos na ausência de informações sobre o estado clínico deles, sendo empregados em estudos de utilização de medicamentos e para fornecer subsídios para estratégias educacionais direcionadas aos profissionais de saúde 6 . Os critérios de avaliação da adequação dos medicamentos utilizados por idosos mais frequentemente observados na literatura são os propostos por Beers et al., publicados em 19912 e atualizados em 1997 3, 20026 e 2012 7, possibilitando contemplar modificações sofridas na farmacoterapia ao longo do tempo.

Evidências sugerem que o uso desses medicamentos é altamente prevalente na população idosa, independente do contexto do cuidado 1,8,9. Adicionalmente, alguns fatores de risco para seu uso, já conhecidos, são de difícil modificação devido, principalmente, à existência de doenças crônicas e de condições resultantes do processo de envelhecimento.

Estudo populacional americano mostrou que $27,9 \%$ dos idosos utilizaram, pelo menos, um medicamento potencialmente inapropriado, sendo que $13,8 \%$ fizeram uso de medicamentos potencialmente inapropriados classificados como alta gravidade, e 14,1\%, de baixa gravidade. Entre os idosos que fizeram uso de medicamentos potencialmente inapropriados, o risco de hospitalização aumentou $20 \%$ 9. Em uma coorte retrospectiva realizada com idosos da comunidade $(\mathrm{N}=17.917$ ), também nos Estados Unidos, verificou-se que $40 \%$ utilizavam, pelo menos, um medicamento potencialmente inapropriado, e $13 \%$, dois ou mais. A ocorrência de problemas relacionados a medicamentos foi maior entre idosos que consumiram medicamentos potencialmente inapropriados $(14,3 \%)$, independente do número, quando comparados àqueles que não faziam uso desses medicamentos $(4,7 \%) 1$. $\mathrm{Na}$ Europa, pesquisa multicêntrica conduzida com idosos admitidos em hospitais mostrou que a prevalência média de uso de medicamentos potencialmente inapropriados, segundo os critérios de Beers, foi de $30,4 \%$, e que a polifarmácia foi preditor do uso desses agentes 10 .

No Brasil, investigações realizadas em diferentes localidades têm apontado variação no uso dos medicamentos potencialmente inapropriados. Estudo de base populacional, realizado em Minas Gerais, apontou a utilização de medicamentos potencialmente inapropriados por $44,7 \%$ dos idosos entrevistados, sendo o gênero feminino significativamente associado ao uso 11. Pesquisa realizada com idosos atendidos pelo Programa Saúde da Família, na Região Nordeste, identificou 34,5\% dos idosos como usuários de medicamentos potencialmente inapropriados, e que os preditores incluíam uso de medicamentos dispensados pelo governo, uso de quatro ou mais medicamentos e aqueles prescritos por médicos 12. Estudo conduzido com idosos de unidades básicas de saúde em Ribeirão Preto, São Paulo, verificou frequência de $48 \%$ de uso de medicamentos potencialmente inapropriados cujos fatores associados foram gênero feminino, automedicação, uso de medicamentos sem prescrição médica, medicamentos psicotrópicos e polifarmácia 13. No âmbito ambulatorial, a prevalência média de medicamentos potencialmente inapropriados identificada a partir de bases de dados de hospital terciário de São Paulo foi de $26,9 \%$, sendo sexo feminino e número de medicamentos prescritos os fatores associados ao uso de medicamentos potencialmente inapropriados 14 .

Considerando os riscos associados ao uso de medicamentos potencialmente inapropriados cujas informações referentes aos idosos da comunidade são relativamente escassas no âmbito nacional, e que o conhecimento da farmacoterapia obtido a partir de estudos populacionais pode auxiliar na elaboração de políticas públicas, o objetivo foi verificar a prevalência e os fatores associados ao uso de medicamentos potencialmente inapropriados, segundo os critérios de Beers, entre as pessoas idosas do Município de São Paulo. 


\section{Método}

Este estudo é parte do Estudo SABE - Saúde, Bem-estar e Envelhecimento, que é um estudo longitudinal, iniciado em 2000, com uma amostra probabilística de idosos (60 anos ou mais) que residem na cidade de São Paulo $(\mathrm{N}=2.143)$ 15. A amostra de referência (2000) foi obtida com um método de amostragem estratificada em dois estágios com base em setores censitários da cidade. Indivíduos com 75 anos ou mais de idade tiveram uma sobreamostra para compensar a maior taxa de mortalidade nessa faixa etária. Mais detalhes sobre o desenho amostral do estudo inicial estão descritos em outra publicação 16.

Em 2006, foram reentrevistados 1.115 idosos, sendo a diferença representada por óbitos (649), recusa (177), não localizados (139), mudanças para outros municípios (51) e institucionalização (12). Nesse mesmo ano, iniciou-se uma nova coorte, composta por 298 indivíduos de 60 a 64 anos, já que essa faixa etária não era mais representada. A amostra transversal final do ano de 2006, então, foi de 1.413. Foram excluídos das análises 155 idosos que não utilizavam medicamentos e quatro idosos por terem ausência de resposta nas variáveis de avaliação funcional. Sendo assim, a amostra do estudo foi composta por 1.254 pessoas, que representavam 903.194 idosos residentes na área urbana do Município de São Paulo, no ano de 2006.

Os critérios de inclusão no estudo foram: idade igual ou superior a 60 anos, pertencer à amostra do Estudo SABE no ano de 2006 e utilizar algum medicamento regularmente.

Os dados foram obtidos por meio de entrevistas domiciliares realizadas por entrevistadores treinados com instrumento padronizado. O questionário foi constituído de seções relativas a vários aspectos da vida do idoso. Os dados físicos e variáveis antropométricas foram coletados por entrevistadores treinados utilizando equipamentos calibrados.

Na coleta dos dados, os 1.254 idosos passaram por uma avaliação cognitiva, e 163, que não foram considerados aptos a responder as questões, tiveram que prosseguir a entrevista com um informante auxiliar ou substituto. Esse informante auxiliar respondeu todas as questões, exceto aquelas de percepção individual, como escala de depressão e outras que não foram utilizadas nas análises deste trabalho.

O Estudo SABE foi aprovado pelo Comitê de Ética em Pesquisa da Faculdade de Saúde Pública, Universidade de São Paulo. Cada entrevistado ou respondente substituto assinou um termo de consentimento livre e esclarecido. Não há conflito de interesses.

\section{Medidas e análises}

O uso dos medicamentos foi determinado a partir das questões "O(a) Sr.(a) poderia me mostrar os remédios que atualmente está usando ou tomando?" e "O(a) Sr.(a) poderia me dizer o nome dos remédios que está usando ou tomando?". Solicitou-se aos entrevistados que mostrassem as embalagens dos medicamentos e a prescrição caso houvesse. Também foram registradas a forma farmacêutica dos medicamentos, a quantidade consumida e o número de tomadas ao dia. Os medicamentos foram classificados de acordo com a classificação Anatomical Therapeutic Chemical (ATC) 17. O uso de medicamentos potencialmente inapropriados foi avaliado segundo os critérios de Beers 6 . A variável dependente do estudo foi então categorizada em "não usa medicamento potencialmente inapropriado" (idosos que não tomavam nenhum medicamento constante na lista de Beers) e "usa medicamento potencialmente inapropriado" (idosos que referiram tomar, pelo menos, um dos medicamentos citados na lista de Beers). O uso de quatro medicamentos não foi considerado, pois sua classificação como medicamentos potencialmente inapropriados depende da dose diária consumida, e essa informação não estava disponível em todos os questionários. São eles: alprazolam (seis idosos), digoxina (34 idosos), lorazepam (11 idosos) e sulfato ferroso (12 idosos). Por outro lado, mesmo na ausência de informação, optou-se por considerar o medicamento nifedipino, mesmo não sabendo se a apresentação utilizada pelos idosos, em 2006, era de "ação rápida". A opção de deixar esse medicamento na análise levou, em consideração, o fato de que, desde a revisão da Relação Nacional de Medicamentos Essenciais (RENAME), publicada em 2007, o nifedipino foi substituído pelo anlodipino como antagonista de canal de cálcio diidropiridínico, com indicação para angina de peito, espasmo coronariano e hipertensão arterial.

Para o cálculo da prevalência, o denominador foi composto por todos os idosos usuários de medicamentos, e o numerador, por aqueles que utilizaram medicamentos potencialmente inapropriados (cada usuário foi considerado uma única vez, independente do número de medicamentos potencialmente inapropriados utilizado).

As análises foram realizadas utilizando variáveis independentes sociodemográficas e de saúde.

- Características demográficas: sexo e idade (categorizada em duas faixas etárias: 60 a 74 anos e 75 anos e mais). A escolaridade foi classificada em anos de estudo e categorizada como analfabeto; 1 a 3 anos de estudo; 4 a 7 anos de estudo 
e 8 ou mais anos de estudo. A renda familiar per capita foi convertida em salários mínimos (até março de 2006, o salário mínimo era equivalente a $\mathrm{R} \$ 300,00$, e, a partir de abril de 2006, o valor considerado foi de $\mathrm{R} \$ 350,00$ ) e categorizada como: sem renda, menor que 1 salário mínimo, 1 a 2,99 salários mínimos, 3 a 4,99 salários mínimos e 5 ou mais salários mínimos. O arranjo familiar foi dividido em vive sozinho e vive acompanhado. Incluiu-se, por fim, o provedor principal de serviços de saúde, classificado em somente público, quando o idoso referiu não ter plano de saúde privado, e privado quando o idoso referiu algum outro plano de saúde.

- Características de saúde: o número de doenças crônicas foi computado a partir das doenças autorreferidas: hipertensão, diabetes, doença pulmonar crônica, doença cardiovascular, acidente vascular cerebral e doença osteoarticular. Foi questionado se o idoso teve episódios de internação no último ano por qualquer motivo e se ocorreram episódios de queda no último ano. O número de medicamentos utilizados foi categorizado entre 1 a 4 medicamentos e 5 ou mais medicamentos (polifarmácia). Adicionalmente, considerou-se declínio cognitivo, atividade funcional e fragilidade. O estado cognitivo foi avaliado por meio da versão modificada do MiniExame do Estado Mental (MEEM), que minimiza os efeitos da escolaridade sobre os resultados do MEEM original 18. A triagem positiva para deficiência cognitiva foi considerada quando o idoso apresentou pontuação inferior a 13 na versão reduzida do MEEM. Nesses casos, aplicouse o questionário desenvolvido por Pfeffer et al. 19, conhecido como Pfeffer Functional Activities Questionnaire (QPAF), destinado a avaliar a autonomia funcional da pessoa no que se refere às atividades de vida quotidiana. Quando o idoso atingiu seis pontos ou mais no QPAF, então foi considerado com deficiência cognitiva. A avaliação funcional foi realizada perguntando-se, para o idoso, se ele apresentava dificuldade em alguma das atividades de vida diária, divididas em atividades básicas (ABVD): atravessar um quarto caminhando, vestir-se, tomar banho, comer, deitar e levantar, usar banheiro; e instrumentais (AIVD): cuidar de dinheiro, usar transporte, fazer compras, usar telefone, tomar medicamentos 20. Foi considerado dependente o idoso que relatou dificuldade em, pelo menos, uma dessas atividades.

A avaliação da síndrome da fragilidade foi identificada pelo fenótipo proposto por Fried et al. 21, composto por cinco componentes: perda de peso não intencional; fadiga autorreferida; fraqueza muscular; baixo nível de atividade física e lentidão na caminhada. Foram considerados não frágeis idosos que não apresentaram nenhum desses componentes; idosos que apresentaram entre um e dois componentes foram classificados como pré-frágeis; e idosos com três ou mais componentes foram considerados frágeis.

Os resultados foram analisados, inicialmente, por meio da análise descritiva da distribuição do uso de medicamentos potencialmente inapropriados. A diferença entre os grupos foi avaliada pelo teste $\chi^{2}$ com a correção de Rao-Scott, que leva, em consideração, os pesos amostrais para estimativas populacionais 22 com nível de significância estabelecido em $5 \%$.

Para a verificação dos fatores associados ao uso de medicamentos potencialmente inapropriados, realizou-se regressão logística univariada relacionando a variável dependente (levando-se, em conta, a categoria de base "não usou medicamento potencialmente inapropriado" e a categoria de interesse "usou medicamento potencialmente inapropriado") com as variáveis independentes de interesse. Foram selecionadas, para o modelo múltiplo, as variáveis que apresentaram $\mathrm{p}<0,20$. Foram mantidas, no modelo final, as variáveis com $\mathrm{p}<0,05$ ou $\mathrm{p}>0,05$, mas que ajustaram alguma outra independente em, pelo menos, $10 \%$.

Todas as análises foram realizadas levandose, em consideração, os pesos amostrais, e as inferências consideraram o efeito de desenho populacional. A análise dos dados foi realizada utilizando o pacote Stata versão 11 (Stata Corp., College Station, Estados Unidos).

\section{Resultados}

A Tabela 1 mostra a distribuição dos idosos segundo características sociodemográficas e uso de medicamentos inapropriados, o qual esteve associado ao sexo feminino e à faixa etária de 75 anos ou mais. A população pesquisada era predominantemente do sexo feminino $(62,6 \%)$ e composta por $74,5 \%$ de indivíduos entre 60 e 74 anos de idade. Quanto à escolaridade, 16,6\% eram analfabetos, 64,1\% tinham entre 1 e 7 anos de estudo, e 19,3\% haviam estudado 8 anos ou mais. Pequena parcela não tinha nenhuma renda (8,3\%), enquanto a maior proporção $(42,1 \%)$ recebia entre 1 e 2,99 salários mínimos da época. A maior parte dos idosos (86,9\%) vivia acompanhada e tinha, como provedor de serviços de saúde, apenas o Sistema Único de Saúde (SUS) $(54,2 \%)$.

Quanto às condições de saúde, 55,3\% referiram ter 2 ou mais doenças. A maioria, 89,5\%, não havia sido internada no ano anterior à coleta dos dados. No mesmo período, 30,4\% sofreram uma ou mais quedas. A maioria, $67 \%$, fazia uso 


\begin{tabular}{|c|c|c|c|c|c|}
\hline \multirow[t]{3}{*}{ Características sociodemográficas } & \multicolumn{2}{|c|}{$\begin{array}{l}\text { Amostra total } \\
(\mathrm{N}=1.254)\end{array}$} & \multicolumn{2}{|c|}{$\begin{array}{c}\text { Uso de medicamentos } \\
\text { potencialmente inapropriados }\end{array}$} & \multirow[t]{3}{*}{ Valor de $p$ * } \\
\hline & $\mathrm{n}$ & $\%$ & $\begin{array}{c}\text { Não } \\
(\mathrm{n}=875)\end{array}$ & $\begin{array}{l}\text { Sim } \\
(n=379)\end{array}$ & \\
\hline & & & $\%$ & $\%$ & \\
\hline Sexo & & & & & $<0,001$ \\
\hline Masculino & 447 & 37,4 & 78,5 & 21,5 & \\
\hline Feminino & 807 & 62,6 & 68,2 & 31,8 & \\
\hline Faixa etária (anos) & & & & & 0,016 \\
\hline $60-74$ & 631 & 74,5 & 73,8 & 26,2 & \\
\hline 75 ou mais & 623 & 25,5 & 66,7 & 33,2 & \\
\hline Escolaridade (anos de estudo) & & & & & 0,308 \\
\hline Analfabeto & 248 & 16,6 & 68,4 & 31,5 & \\
\hline $1-3$ & 345 & 25,8 & 75,9 & 24,1 & \\
\hline $4-7$ & 450 & 38,3 & 71,0 & 29,0 & \\
\hline 8 ou mais & 208 & 19,3 & 72,1 & 27,9 & \\
\hline Renda em salários mínimos ** & & & & & 0,108 \\
\hline Sem renda & 81 & 8,3 & 65,2 & 34,8 & \\
\hline$<1$ & 265 & 20,2 & 69,6 & 30,4 & \\
\hline $1-2,99$ & 502 & 42,1 & 69,3 & 30,7 & \\
\hline $3-4,99$ & 167 & 15,1 & 77,4 & 22,6 & \\
\hline 5 ou mais & 153 & 14,3 & 78,1 & 21,9 & \\
\hline Arranjo familiar & & & & & 0,199 \\
\hline Vive sozinho & 198 & 13,1 & 76,3 & 23,7 & \\
\hline Vive acompanhado & 1.053 & 86,9 & 71,3 & 28,7 & \\
\hline Provedor de serviços de saúde & & & & & 0,844 \\
\hline Somente público & 666 & 54,2 & 71,8 & 28,2 & \\
\hline Privado & 588 & 45,8 & 72,3 & 27,6 & \\
\hline
\end{tabular}

* Diferenças entre grupos testadas utilizando teste $\chi^{2}$ com correção de Rao-Scott;

** Valor do salário mínimo até março de $2006=\mathrm{R} \$ 300,00$; a partir de abril de $2006=\mathrm{R} \$ 350,00$.

de 1 a 4 medicamentos. 75,4\% não apresentavam dificuldades com as atividades básicas de vida diária, enquanto 64,4\% não tinham dificuldades com as atividades instrumentais de vida diária. Ainda, 71,5\% não apresentavam deficiência cognitiva. Com relação à fragilidade, $14 \%$ foram classificados como frágeis; $38,5 \%$, como não frágeis; e $47,5 \%$ foram considerados pré-frágeis, como mostra a Tabela 2. Na mesma tabela, verifica-se que as condições de saúde que se mostraram associadas ao uso de medicamentos potencialmente inapropriados foram a presença de 2 ou mais doenças, o uso de 5 medicamentos ou mais, dificuldades com as ABVD e com as AIVD e síndrome de fragilidade.

Na análise, pelo modelo de regressão logística múltipla, as variáveis associadas ao consumo de medicamentos potencialmente inapropriados foram uso de 5 medicamentos ou mais $(\mathrm{p}=$ $0,001)$, presença de 2 ou mais doenças $(p=0,011)$ e sexo feminino $(p=0,007)$, ajustadas por fragilidade, idade e dificuldade em ABVD (Tabela 3).

Constatou-se uma prevalência de $28 \%$ (IC95\%: 25,3; 30,7) de uso de medicamentos potencialmente inapropriados entre os idosos do Município de São Paulo, em 2006. Dentre esses, $83,8 \%$ utilizavam um único medicamento potencialmente inapropriado, $13,8 \%$ usavam dois, enquanto $2,4 \%$ usavam de 3 até 5 .

A frequência proporcional de cada medicamento potencialmente inapropriado usado pelos idosos participantes do Estudo SABE, em 2006, está listada na Tabela 4. Os medicamentos potencialmente inapropriados mais utilizados 
Distribuição proporcional dos idosos segundo as condições de saúde e uso de medicamentos potencialmente inapropriados ( $N=1.254$ idosos). Município de São Paulo, Brasil, Estudo SABE, 2006.

\begin{tabular}{|c|c|c|c|c|c|}
\hline \multirow[t]{3}{*}{ Condições de saúde } & \multicolumn{2}{|c|}{$\begin{array}{l}\text { Amostra total } \\
(N=1.254)\end{array}$} & \multicolumn{2}{|c|}{$\begin{array}{c}\text { Uso de medicamentos } \\
\text { potencialmente inapropriados }\end{array}$} & \multirow[t]{3}{*}{ Valor de $p$ * } \\
\hline & $\mathrm{n}$ & $\%$ & $\begin{array}{c}\text { Não } \\
(n=875)\end{array}$ & $\begin{array}{c}\text { Sim } \\
(n=379)\end{array}$ & \\
\hline & & & $\%$ & $\%$ & \\
\hline Número de doenças crônicas & & & & & $<0,001$ \\
\hline Nenhuma ou 1 & 528 & 44,7 & 79,8 & 20,2 & \\
\hline 2 ou mais & 726 & 55,3 & 65,8 & 34,2 & \\
\hline Internação no último ano & & & & & 0,576 \\
\hline Nenhuma & 1.095 & 89,5 & 72,3 & 27,7 & \\
\hline Uma ou mais & 159 & 10,5 & 69,9 & 30,1 & \\
\hline Queda no último ano & & & & & 0,299 \\
\hline Nenhuma & 836 & 69,6 & 73,1 & 26,9 & \\
\hline Uma ou mais & 418 & 30,4 & 69,7 & 30,3 & \\
\hline Número de medicamentos & & & & & $<0,001$ \\
\hline $1-4$ & 799 & 67,0 & 77,9 & 22,1 & \\
\hline 5 ou mais & 455 & 33,0 & 60,0 & 40,0 & \\
\hline Deficiência cognitiva & & & & & 0,010 \\
\hline Não & 816 & 71,5 & 74,2 & 25,8 & \\
\hline Sim & 438 & 28,5 & 66,6 & 33,4 & \\
\hline Dificuldade em ABVD & & & & & $<0,001$ \\
\hline Não & 855 & 75,4 & 75,5 & 24,5 & \\
\hline Sim & 399 & 24,6 & 61,1 & 38,8 & \\
\hline Dificuldade em AIVD & & & & & $<0,001$ \\
\hline Não & 683 & 64,4 & 75,8 & 24,2 & \\
\hline Sim & 571 & 35,6 & 65,4 & 34,6 & \\
\hline Síndrome de fragilidade & & & & & 0,001 \\
\hline Não frágil & 392 & 38,5 & 76,3 & 23,7 & \\
\hline Pré-frágil & 600 & 47,5 & 72,2 & 27,8 & \\
\hline Frágil & 262 & 14,0 & 59,9 & 40,1 & \\
\hline
\end{tabular}

ABVD: atividades básicas de vida diária; AIVD: atividades instrumentais de vida diária.

* Diferenças entre grupos testadas utilizando teste $\chi^{2}$ com correção de Rao-Scott.

foram aqueles que atuam no sistema cardiovascular (10,3\%), destacando-se o nifedipino (3,7\%), a amiodarona $(3,2 \%)$ e a metildopa $(3 \%)$. O segundo grupo mais utilizado foi o de medicamentos que atuam no sistema nervoso central $(7,2 \%)$, destacando-se a amitriptilina (2,3\%). Os medicamentos que atuam no sistema musculoesquelético representaram 5,7\% dos medicamentos potencialmente inapropriados utilizados, e, desses, o mais utilizado foi a orfenadrina $(2,6 \%)$.

\section{Discussão}

Os achados mostram que mais de um quarto dos idosos do Município de São Paulo foram consumidores de medicamentos potencialmente inapropriados. Dentre esses, 13,8\% utilizavam dois medicamentos potencialmente inapropriados, e alguns utilizavam até 5 desses medicamentos, com consequências imprevisíveis de seus efeitos, apesar das evidências acerca dos riscos e impacto negativo relativo ao seu uso. Revisão sistemática demonstrou que o uso de medicamentos potencialmente inapropriados tem sido associado com reações adversas e hospitalização em idosos provenientes, respectivamente, 
Fatores associados com o uso de medicamentos potencialmente inapropriados por idosos residentes no Município de São Paulo, Brasil, após análise de regressão múltipla ( $N=1.254$ idosos). Estudo SABE, 2006.

\begin{tabular}{lcc}
\hline Variáveis & Análise bruta & Análise ajustada \\
& OR (IC95\%) & OR (IC95\%) \\
\hline Uso de 5 medicamentos ou mais & $2,35(1,75 ; 3,15)$ & $1,78(1,28 ; 2,47)$ \\
Fragilidade & 1,00 & 1,00 \\
$\quad$ Não frágil & $1,24(0,89 ; 1,71)$ & $1,04(0,74 ; 1,45)$ \\
$\quad$ Pré-frágil & $2,15(1,50 ; 3,08)$ & $1,41(0,89 ; 2,25)$ \\
$\quad$ Frágil & $2,05(1,54 ; 2,72)$ & $1,50(1,10 ; 2,04)$ \\
Presença de 2 ou mais doenças crônicas & $1,96(1,45 ; 2,65)$ & $1,27(0,89 ; 1,80)$ \\
Dificuldade em ABVD & $1,70(1,27 ; 2,28)$ & $1,53(1,12 ; 2,07)$ \\
Sexo feminino & $1,41(1,07 ; 1,85)$ & $1,12(0,80 ; 1,55)$ \\
Idade: 75 anos ou mais &
\end{tabular}

ABVD: atividades básicas de vida diária; IC95\%: intervalo de 95\% de confiança; OR: odds ratio.

de diferentes contextos assistenciais e da comunidade 23. Metanálise que totalizou 90.611 participantes apontou que idosos que utilizaram medicamentos potencialmente inapropriados apresentaram maior risco para mortalidade, independente do cenário analisado, da existência de comorbidades, de polifarmácia ou do tipo de medicamento potencialmente inapropriado usado 24 . No contexto nacional, pesquisa realizada com idosos hospitalizados constatou que o uso de um medicamento potencialmente inapropriado dobrou a chance de o paciente apresentar uma reação adversa 25.

A ocorrência dessas adversidades afeta não apenas o idoso, mas, também, a sociedade e os sistemas de saúde, implicando na necessidade da implementação de políticas públicas 23,24 .

Investigações prévias relataram amplo uso de medicamentos potencialmente inapropriados em instituições de longa permanência, ambulatórios, hospitais e comunidade 1,10,11,12,13,14,25,26, com prevalências que variaram de $10,4 \% 26$ a $48 \% 13$. Estudo multicêntrico, realizado em seis países europeus, evidenciou prevalência média de $30,4 \% 10$, e outro, norte-americano, mostrou que $27,9 \%$ dos idosos utilizaram medicamentos potencialmente inapropriados 1. Em ambos, a frequência de uso foi bastante semelhante ao do presente estudo, a despeito das diferenças no que tange, especialmente, aos formulários de medicamentos nacionais e à lista de medicamentos vendidos sem prescrição médica nesses países.

No âmbito nacional, também se observa uma grande variação na frequência do uso de medicamentos potencialmente inapropriados entre idosos da comunidade que utilizaram a mesma versão dos critérios de Beers. Assim, entre idosos aposentados, vivendo no Rio de Janeiro, observou-se uma baixa frequência de uso de medicamentos potencialmente inapropriados de $10,4 \%$, o que pode ter ocorrido por tratar-se, segundo os próprios autores, de estudo exploratório 26. Por outro lado, em Diamantina, Minas Gerais, a frequência de uso de medicamentos potencialmente inapropriados foi de $44,73 \%$. Nesse caso, a amostra do estudo foi determinada para conhecer o perfil de utilização de medicamentos por indivíduos maiores de 18 anos, e 21,5\% da amostra se referia a indivíduos de 60 anos ou mais 11 . Outro estudo, realizado com idosos usuários de unidades básicas de saúde, apontou frequência de uso de $48 \%$. A utilização de medicamentos potencialmente inapropriados bem superior à do presente estudo pode ser devida ao fato de $30 \%$ daqueles idosos praticarem a automedicação, e de $82,3 \%$ dos usuários de medicamentos potencialmente inapropriados apresentarem de 60 a 75 anos 13. No SABE, os idosos que usaram medicamentos potencialmente inapropriados, nessa mesma faixa etária, representaram $26,2 \%$ da população estudada. A variação nos valores de prevalência pode se dar pelos distintos delineamentos dos estudos, que apresentam amostras de tamanhos diversos e em diferentes contextos, além do perfil clínico e demográfico dos idosos e do cenário da assistência farmacêutica em cada localidade.

Nesta pesquisa, o uso de 5 ou mais medicamentos e a presença de 2 ou mais doenças crônicas estiveram associados ao uso de medicamentos potencialmente inapropriados. $\mathrm{O}$ uso de vários medicamentos tem se mostrado asso- 
Distribuição proporcional dos medicamentos potencialmente inapropriados segundo grupo anatômico do sistema Anatomical Therapeutic Clinical (ATC) utilizados * por idosos. Município de São Paulo, Brasil, Estudo SABE, 2006.

\begin{tabular}{|c|c|}
\hline $\begin{array}{l}\text { Sistema (fração de ocorrência)/Medicamentos } \\
\text { potencialmente inapropriados }\end{array}$ & $\%$ \\
\hline \multicolumn{2}{|l|}{ Cardiovascular (10,3\%) } \\
\hline Nifedipino & 3,7 \\
\hline Amiodarona & 3,2 \\
\hline Metildopa & 3,0 \\
\hline Doxazosina & 0,8 \\
\hline Codergocrina (mesilato de) & 0,2 \\
\hline Clonidina & 0,2 \\
\hline \multicolumn{2}{|l|}{ Nervoso central $(7,2 \%)$} \\
\hline Amitriptilina & 2,3 \\
\hline Diazepam & 2,0 \\
\hline Fluoxetina & 1,8 \\
\hline Hidroxizine & 0,7 \\
\hline Clordiazepóxido & 0,4 \\
\hline Anoréxicos & 0,4 \\
\hline Clordiazepóxido + Amitriptilina & 0,1 \\
\hline Anfetaminas & 0,1 \\
\hline Barbitúricos & 0,1 \\
\hline Flurazepam & 0,1 \\
\hline \multicolumn{2}{|l|}{ Musculoesquelético (5,7\%) } \\
\hline Orfenadrina & 2,6 \\
\hline Carisoprodol & 1,5 \\
\hline Piroxicam & 0,8 \\
\hline Naproxeno & 0,4 \\
\hline Cetorolaco & 0,3 \\
\hline Ciclobenzaprina & 0,1 \\
\hline \multicolumn{2}{|l|}{ Digestivo e metabolismo $(3,6 \%)$} \\
\hline Clorpropamida & 1,2 \\
\hline Belladonna (alcaloides da) & 1,1 \\
\hline Óleo mineral & 0,7 \\
\hline Cimetidina & 0,4 \\
\hline Bisacodil & 0,2 \\
\hline \multicolumn{2}{|l|}{ Respiratório (2,3\%) } \\
\hline Dexclorfeniramina & 1,5 \\
\hline Difenidramina & 0,5 \\
\hline Prometazina & 0,3 \\
\hline \multicolumn{2}{|l|}{ Geniturinário (1,7\%) } \\
\hline Estrógenos & 0,8 \\
\hline Propantelina & 0,5 \\
\hline Oxibutinina & 0,4 \\
\hline \multicolumn{2}{|l|}{ Sangue $(0,8 \%)$} \\
\hline Ticlopidina & 0,5 \\
\hline Dipiridamol & 0,3 \\
\hline \multicolumn{2}{|l|}{ Antibacterianos para uso sistêmico $(0,2 \%)$} \\
\hline Nitrofurantoína & 0,2 \\
\hline
\end{tabular}

* Alguns idosos utilizaram mais de um medicamento potencialmente inapropriado. ciado ao uso de medicamentos potencialmente inapropriados em vários estudos, realizados no Brasil e em outros países 10,12,13,27,28,29,30. Revisão sistemática recente apontou a polifarmácia como a variável mais fortemente associada à utilização de medicamentos potencialmente inapropriados, afirmando que o uso de vários medicamentos pode expor os idosos a substâncias cujos riscos superam os benefícios 31 . Outros autores 10,26,32 apontam, também, que o risco de uso de medicamentos potencialmente inapropriados é maior entre os indivíduos com maior necessidade de medicamentos, ou seja, os que apresentam maior número de morbidades, como observado no presente estudo.

O sexo feminino também esteve associado ao uso de medicamentos potencialmente inapropriados, como em outros trabalhos 13,28, e já havia sido apontado como fator associado na revisão já citada 31 . O sexo feminino representou $62,6 \%$ da população pesquisada. De maneira geral, mulheres utilizam mais medicamentos que homens. As razões do uso de medicamentos predominantemente entre idosas parecem estar ligadas a questões de ordem biológica, já que são mais expostas a problemas de saúde não fatais. Mulheres são mais preocupadas quanto aos sintomas físicos e psicológicos, colocando maior atenção aos problemas de saúde. Há também aspectos socioculturais, uma vez que, ao longo da vida, utilizam mais frequentemente os serviços de saúde e estão mais familiarizadas com os medicamentos 28,31,33.

As variáveis que indicam as condições de saúde referentes ao declínio cognitivo, ao desempenho físico, avaliado como ABVD e AIVD, e à fragilidade não figuraram como significantes na regressão logística múltipla. Entretanto, é importante destacar que essas variáveis apresentaram-se associadas ao uso de medicamentos potencialmente inapropriados na análise univariada. Esse reconhecimento, analisado à luz da prática clínica, é importante, porque o monitoramento de usuários de medicamentos potencialmente inapropriados pode evitar ou reduzir agravos à saúde envolvidos com a perda de habilidades funcionais dos idosos, com o declínio cognitivo e com a síndrome da fragilidade.

$\mathrm{Na}$ análise dos medicamentos potencialmente inapropriados agrupados por sistema ATC, observa-se que o de maior frequência de uso foi o grupo de medicamentos que atuam no sistema cardiovascular (10,3\%). Esse fato não surpreende, dado que diversos estudos populacionais brasileiros identificaram essa classe de medicamentos como a mais utilizada por idosos $33,34,35,36$. As doenças que afetam o sistema cardiovascular são as mais prevalentes nesse grupo 
etário 37 , conforme verificado no grupo estudado, no qual $68,3 \%$ dos entrevistados referiram hipertensão (Departamento de Informática do SUS. Indicadores e Dados Básicos. http://www2. datasus.gov.br, acessado em 07/Fev/2011).

$\mathrm{O}$ nifedipino foi o medicamento mais utilizado $(3,7 \%)$ pelos idosos estudados. A formulação do nifedipino de "ação rápida" é classificada como inadequada, pelos critérios de Beers, pelo risco potencial para hipotensão e constipação, sendo as possíveis consequências do uso consideradas de alta gravidade. Outro medicamento potencialmente inapropriado incluído neste grupo foi o agente antiarrítmico amiodarona (3,2\%). Não há muita informação clínica e farmacocinética de seu uso em idosos, entretanto, parece predispor esse grupo etário à toxicidade. Assim, seu uso por idosos requer o acompanhamento dos pacientes, recomendando-se o início do tratamento com dosagem mínima ${ }^{38}$.

O terceiro medicamento potencialmente inapropriado mais utilizado (3\%) também atua no sistema cardiovascular, o inibidor alfa-adrenérgico metildopa. Em decorrência dos efeitos no sistema nervoso central, como bradicardia e exacerbação de depressão, a metildopa não é considerada um medicamento de primeira escolha para tratar hipertensão em idosos, sendo necessário, inclusive, ajuste de dose dependente da função renal ${ }^{38}$. O uso desses medicamentos foi relatado em outros inquéritos nacionais $12,26 \mathrm{e}$ em revisão sistemática ${ }^{24}$, mostrando que, apesar das evidências e dos riscos superarem os benefícios, prescritores continuam indicando esses agentes, sugerindo a necessidade de mudanças nos padrões de prescrição em nível mundial.

No agrupamento dos medicamentos potencialmente inapropriados com ação no sistema nervoso central $(7,2 \%)$, foram identificados benzodiazepínicos como clordiazepóxido, clordiazepóxido + amitriptilina e diazepam. Esses fármacos têm meia-vida longa em pacientes idosos (geralmente vários dias), produzindo sedação prolongada e aumentando o risco de quedas e fraturas, consequências consideradas de alta gravidade. Ainda, podem causar confusão e delírio, que podem ser erroneamente confundidos com demência primária 39,40 . A frequência expressiva de uso de diazepam relatada em estudos brasileiros, incluindo o SABE, pode estar relacionado ao seu baixo custo e disponibilidade, dado que é distribuído pelo SUS. Além disso, é um dos benzodiazepínicos mais frequentemente prescritos para tratar a insônia, condição frequentemente observada em idosos, devido à alteração do sono 41.

Dos antidepressivos, foram identificados a amitriptilina e a fluoxetina. Por causa das suas propriedades anticolinérgicas e forte sedação, a amitriptilina raramente é o antidepressivo de escolha para pacientes idosos, sendo as consequências de seu uso consideradas de alta gravidade. A fluoxetina tem meia-vida longa e apresenta risco de produzir estimulação excessiva do sistema nervoso central, alterações do sono e agitação crescente, sendo considerado de alta gravidade usá-la "diariamente" 38 .

O terceiro grupo de medicamentos potencialmente inapropriados mais utilizados foi o de medicamentos que atuam no sistema musculoesquelético $(5,7 \%)$, no qual houve predomínio de cetorolaco, naproxeno e piroxicam. Esses fármacos têm potencial para produzir sangramento digestivo, aumento da pressão arterial e insuficiência renal e cardíaca 6 . A maioria dos relaxantes musculares e medicamentos antiespasmódicos é mal tolerada pelos pacientes idosos, pois causam efeitos anticolinérgicos 42 , além de sedação e fraqueza. Adicionalmente, a eficácia terapêutica desses agentes pelos pacientes idosos é questionável 6 .

O fármaco orfenadrina, também identificado, em geral, é produzido em associação à dipirona e cafeína, e o carisoprodol costuma ser produzido em comprimidos associados a paracetamol, diclofenaco e cafeína. Esses medicamentos são comercializados no Brasil sem necessidade de prescrição, possibilitando sua utilização como automedicação.

No que tange aos medicamentos com propriedades sedativas e anticolinérgicas, a ferramenta chamada "índice de carga de medicamentos" (drug burden index - DBI) mede o peso da exposição total do idoso a medicamentos como os alcaloides naturais atropina e escopolamina 43,44 , que foram encontrados neste estudo, embora com baixa frequência de uso. O DBI demonstrou que a exposição a esses medicamentos está associada a pior desempenho físico e cognitivo em idosos. No SABE, as condições de saúde referentes ao declínio cognitivo e ao desempenho físico dos idosos pesquisados mostraram associação positiva com o uso de medicamentos potencialmente inapropriados. Entretanto, por se tratar de estudo transversal, a relação causa e efeito entre os eventos não foi detectável. Assim, o surgimento de incapacidades pode ter levado ao uso de medicamentos potencialmente inapropriados, agravando a condição de saúde dos idosos participantes, mas, inversamente, o uso de medicamentos potencialmente inapropriados pode ter levado a incapacidades.

Este estudo apresenta algumas limitações. A prevalência de utilização de medicamentos potencialmente inapropriados pode ter sido subestimada, uma vez que foram pesquisados apenas 
os medicamentos listados pelos critérios de Beers. Não foram considerados aqueles tidos como medicamentos potencialmente inapropriados apenas na presença de certas doenças ou condições e aqueles classificados como medicamentos potencialmente inapropriados em função da dose diária consumida. Nesse caso, frequentemente a prevalência é menor do que quando aplicados os critérios completos 45. Desse modo, devem ser realizados outros estudos, especialmente de base populacional, que possam confirmar ou refutar os achados dessa investigação, de maneira a testar, medir e adequar instrumento(s) para melhor compreensão desse problema de saúde pública dos idosos brasileiros.

Sugere-se, para a realidade nacional, a elaboração de método baseado em critérios explícitos de avaliação da farmacoterapia em idosos, levando-se, em consideração, os medicamentos disponíveis no país, a opinião de especialistas brasileiros e a literatura baseada em evidências na área de utilização de medicamentos.

A definição de medicamentos potencialmente inapropriados por listas específicas, em vez de outros mecanismos (tais como o seguimento farmacoterapêutico do paciente), pode não detectar alguns problemas, tais como a subutilização, a falta de adesão ao tratamento e as interações de medicamentos. Ainda, tendo em vista as limitações dos critérios presentes na literatura e a he- terogeneidade da população idosa, o julgamento clínico deve ser sempre considerado na tomada de decisões em relação à farmacoterapia do idoso. De qualquer forma, a aplicação de critérios explícitos na prática clínica para identificação de problemas e adequação do uso de medicamentos pode auxiliar equipes multidisciplinares de saúde, melhorando a qualidade do cuidado.

A maioria dos medicamentos potencialmente inapropriados identificados neste estudo requer prescrição médica, alguns, inclusive, com retenção de receita no momento da compra. Tal fato aponta para a necessidade de cuidado na prescrição, o que pode ser incentivado por meio de intervenções educativas, com ampla divulgação dos aspectos farmacoterapêuticos relativos ao idoso. Além disso, o uso de protocolos ou softwares para prescrição podem ser úteis para orientar a farmacoterapia desse grupo etário.

Concluindo, apesar dos riscos associados ao uso de medicamentos potencialmente inapropriados, verifica-se que eles são amplamente utilizados pelos idosos do Município de São Paulo. $\mathrm{O}$ risco associado ao uso por mulheres, pessoas com duas ou mais doenças ou que usam cinco ou mais medicamentos faz com que esses sejam grupos que merecem atenção especial, dado o potencial de sofrerem eventos adversos graves, com consequências negativas aos idosos e ao sistema de saúde.

\section{Resumen}

Algunos medicamentos son considerados inapropiados para los ancianos, debido a alteraciones en la farmacocinética y en la farmacodinámica, por falta de eficacia terapéutica o por un riesgo de aumento de efectos adversos, superando sus beneficios. Se verificó la prevalencia del uso de medicamentos potencialmente inapropiados, según los criterios de Beers, por parte de ancianos del municipio de São Paulo, Brasil, participantes en el estudio SABE. Se trata de un estudio transversal de base poblacional, cuya muestra de 1.254 individuos con 60 años o más representaba a la población anciana en el municipio durante el año 2006. La prevalencia de uso de medicamentos potencialmente inapropiados fue de un $28 \%$. En el modelo de regresión logística múltiple, las variables asociadas al uso fueron polifarmacia ( $p=0,001)$, dos o más enfermedades $(p=0,011)$ y sexo femenino $(p=0,007)$. Se identificó el uso de 36 medicamentos potencialmente inapropiados, la mayoría de venta con prescripción. Son necesarios criterios específicos para la población brasileña y una amplia divulgación de la farmacología clínica del anciano. Protocolos o softwares para prescripción pueden auxiliar en la prescripción racional para este grupo.

Utilización de Medicamentos; Anciano; Farmacoepidemiología 


\section{Colaboradores}

T. C. J. Cassoni e N. S. Romano-Lieber participaram da concepção do projeto, da análise e interpretação dos dados e da redação do artigo; L. P. Corona e S. R. Secoli colaboraram na análise e interpretação dos dados e da redação do artigo. Y. A. O. Duarte e M. L. Lebrão contribuíram na concepção do projeto e na análise e interpretação dos dados. Todas as autoras participaram da revisão crítica para publicação do artigo e aprovaram a versão final encaminhada.

\section{Referências}

1. Fick DM, Mion LC, Beers MH, Waller JL. Health Outcomes associated with potentially inappropriate medication use in older adults. Res Nurs Health 2008; 31:42-51.

2. Beers MH, Ouslander JG, Pollingher J, Reuben DB, Brooks J, Beck JC. Explicit criteria for determining inappropriate medication use in nursing homes residents. Arch Intern Med 1991; 151:1825-32.

3. Beers MH. Explicit criteria for determining potentially inappropriate medication use by the elderly: an update. Arch Int Med 1997; 157:1531-6.

4. Nóbrega OT, Karnikowski MG. A terapia medicamentosa no idoso: cuidados na medicação. Ciênc Saúde Coletiva 2005; 10:309-12.

5. Ribeiro $\mathrm{AQ}$, Araújo $\mathrm{CM}$, Acurcio $\mathrm{FA}$, Magalhães SM, Chaimowicz F. Qualidade do uso de medicamentos por idosos: uma revisão dos métodos de avaliação disponíveis. Ciênc Saúde Coletiva 2005; 10:1037-45.

6. Fick DM, Cooper JW, Wade WE, Waller JL, Maclean JR, Beers MH. Updating the Beers criteria for potentially inappropriate medication use in older adults. Arch Intern Med 2003; 163:2716-24.

7. American Geriatrics Society 2012 Beers Criteria Update Expert Panel. American Geriatrics Society updated Beers criteria for potentially inappropriate medication use in older adults. J Am Geriatr Soc 2012; 60:616-31.
Agradecimentos

À FAPESP (processo 1999/05125-7) pelo financiamento.
8. Klarin I, Wimo A, Fastbom J. The association of inappropriate drug use with hospitalization and mortality: a population-based study of the very old. Drugs Aging 2005; 22:69-82.

9. Fillenbaun GG, Hanlon JT, Landerman LR, Artz M, O'Connor H, Dowd B, et al. Impact of inappropriate drug use on health services utilization among representative older community-dwelling residents. Am J Geriatr Pharmacother 2004; 2:92-101.

10. Gallagher P, Lang PO, Cherubini A, Topinková E, Cruz-Jentoft A, Errasquin BM, et al. Prevalence of potentially inappropriate prescribing in an acutely ill population of older patients admitted to six European hospitals. Eur J Clin Pharmacol 2011; 67:1175-88.

11. Pinto MCX, Ferré F, Pinheiro MLP. Potentially inappropriate medication use in a city of Southeast Brazil. Braz J Pharm Sci 2012; 48:79-86.

12. Oliveira MG, Amorim WW, Jesus SR, Rodrigues VA, Passos LC. Factors associated with potentialy inappropriate medication use by the elderly in the Brazilian primary care setting. Int J Clin Pharm 2012; 34:626-32.

13. Baldoni AD, Ayres LR, Martinez EZ, Dewulf ND, Santos V, Pereira LR. Factors associated with potentially inappropriate medications use by the elderly according to Beers criteria 2003 and 2012. Int J Clin Pharm 2013; [Epub ahead of print]. 
14. Faustino CG, Passarelli MCG, Jacob-Filho W. Potentially inappropriate medications among elderly Brazilian outpatients. São Paulo Med J 2013; 31:19-26.

15. Lebrão ML. O projeto SABE em São Paulo: uma visão panorâmica. In: Lebrão ML, Duarte YAO, organizadores. O projeto SABE no Município de São Paulo: uma abordagem inicial. Brasília: Organização Pan-Americana da Saúde; 2003. p. 33-44.

16. Silva N. Aspectos metodológicos - processo de amostragem. In: Lebrão ML, Duarte YAO, organizadores. O projeto SABE no Município de São Paulo: uma abordagem inicial. Brasília: Organização Pan-Americana da Saúde; 2003. p. 47-58.

17. WHO Collaborating Centre for Drug Statistics Methodology. Anatomical Therapeutic Chemical (ATC) classification index with defined daily doses (DDDs). http://www.whocc.no/atc_ddd_index/ (acessado em 28/Fev/2013).

18. Folstein MF, Folstein SE, McHugh PR. "Mini-mental state": a practical method for grading the cognitive state of patients for the clinician. J Psychiatr Res 1975; 12:189-98.

19. Pfeffer RI, Kurosaki TT, Harrah Jr. CH, Chance JM, Filos S. Measurement of functional activities in older adults in the community. J Gerontol 1987 37:323-9.

20. Duarte YAO. Desempenho funcional e demandas assistenciais. In: Lebrão ML, Duarte YAO, organizadores. O projeto SABE no Município de São Paulo: uma abordagem inicial. Brasília: Organização Pan-Americana da Saúde; 2003. p. 185-200.

21. Fried LP, Tangen CM, Walston J, Newman AB, Hirsch C, Gottdiener J, et al. Frailty in older adults: evidence for a phenotype. J Gerontol A Biol Sci Med Sci 2001; 56A:M146-56.

22. Rao JNK, Scott AJ. On simple adjustments to chisquare tests with sample survey data. Ann Stat 1987; 15:385-97.

23. Jano E, Aparasu R. Healthcare outcomes associated with Beers's criteria: a systematic review. Geriatrics 2007; 41:438-48.

24. Sichieri K, Rodrigues ARB, Takahashi JA, Secoli SR, Nobre MRC, Martinez MA, et al. Mortality associated with of the use of inappropriate drugs according Beers criteria: a systematic review. Advances in Pharmacology and Pharmacy 2013; 1:74-84.

25. Passarelli MCG, Jacob-Filho W, Figueras A. Adverse drug reactions in an elderly hospitalised population. Drugs Aging 2005; 22:767-77.

26. Rozenfeld S, Fonseca MJM, Acurcio FA. Drug utilization and polypharmacy among the elderly: a survey in Rio de Janeiro City, Brazil. Rev Panam Salud Pública 2008; 23:34-43.

27. Buck MD, Atreja A, Brunker CP, Jain A, Suh TT, Palmer RM, et al. Potentially inappropriate medication prescribing in outpatient practices: prevalence and patient characteristics based on electronic health records. Am J Geriatr Pharmacother 2009; 7:84-92.
28. Gavilán Moral E, Suárez-Varela MT, Hoyos Esteban JA, Pérez Suanes AM. Polimedicación y prescripción de fármacos inadecuados en pacientes ancianos inmovilizados que viven en la comunidad. Aten Primaria 2006; 38:476-82.

29. Holguín-Hernández EH, Orozco-Diaz JG. Medicación potencialmente inapropiada en ancianos en un hospital de primer nivel, Bogotá 2007. Rev Salud Pública 2010; 12:287-99.

30. Gallagher PF, Barry PJ, Ryan C, Hartigan I, O'Mahony D. Inappropriate prescribing in an acutely ill population of elderly patients as determined by Beers' criteria. Age Ageing 2008; 37: 96-101.

31. Guaraldo L, Cano FG, Damasceno GS, Rozenfeld S. Inappropriate medication use among the elderly: a systematic review of administrative databases. BMC Geriatr 2011; 11:79.

32. Blalock SJ, Byrd JE, Hansen RA, Yamanis TJ, McMullin K, DeVellis BM, et al. Factors associated with potentially inappropriate drug utilization in a sample of rural community-dwelling older adults. Am J Geriatr Pharmacother 2005; 3:168-79.

33. Loyola Filho AI, Uchoa E, Lima-Costa MF. Estudo epidemiológico de base populacional sobre uso de medicamentos entre idosos na Região Metropolitana de Belo Horizonte, Minas Gerais, Brasil. Cad Saúde Pública 2006; 22:2657-67.

34. Lima MG. Fatores associados aos gastos com medicamentos utilizados por aposentados e pensionistas idosos em Belo Horizonte/MG [Dissertação de Mestrado]. Belo Horizonte: Faculdade de Farmácia, Universidade Federal de Minas Gerais; 2008.

35. Flores LM, Mengue SS. Uso de medicamentos por idosos em região do sul do Brasil. Rev Saúde Pública 2005 ; 39:924-9.

36. Coelho Filho JM, Marcopito LF, Castelo A. Perfil de utilização de medicamentos por idosos em área urbana do Nordeste do Brasil. Rev Saúde Pública 2004; 38:557-64.

37. Organização Mundial da Saúde. Envelhecimento ativo: uma política de saúde. Brasília: Organização Pan-Americana da Saúde; 2005.

38. Semla T, Beizer JL, Higbee MD. Geriatric dosage handbook: including clinical recommendations and monitoring guidelines. 13th Ed. Hudson: LexiComp; 2008.

39. Wortelboer U, Cohrs S, Rodenbeck A, Rüther El. Tolerability of hypnosedatives in older patients. Drugs Aging 2002; 19:529-39.

40. Montgomery P, Dennis JA. Physical exercise for sleep problems in adults aged $60+$. Cochrane Database Syst Rev 2002; (4):CD003404.

41. Noia AS, Secoli SR, Duarte YA, Lebrão ML, Romano-Lieber NS. Factors associated to the use of psychotropc drugs by community-dwelling elderly in São Paulo City. Rev Esc Enferm USP 2012; 46(n. spe):38-43. 
42. Ness J, Hoth A, Barnett MJ, Shorr RI, Kaboli PJ. Anticholinergic medications in community-dwelling older veterans: prevalence of anticholinergic symptoms, symptom burden, and adverse drug events. Am J Geriatr Pharmacother 2006; 4:42-51.

43. Hilmer SN, Mager DE, Simonsick EM, Cao Y, Ling SM, Windham BG, et al. A drug burden index to define the functional burden of medications in older people. Arch Intern Med 2007; 167:781-7.

44. Brown HJ, Taylor P. Agonistas e antagonistas dos receptores muscarínicos. In: Brunton LL, Lazo JS, Parker KL, organizadores. Goodman \& Gilman: as bases farmacológicas da terapêutica. 11a Ed. Rio de Janeiro: McGraw-Hill Interamericana do Brasil; 2006. p. 165-81.
45. Chang CB, Chan CD. Comparison of published explicit criteria for potentially inappropriate medications in older adults. Drugs Aging 2010; 27:947-57.

Recebido em 11/Mar/2013

Versão final reapresentada em 24/Jan/2014

Aprovado em 07/Fev/2014 